Check for updates

Cite this: RSC Adv., 2018, 8, 21841

Received 17th May 2018

Accepted 6th June 2018

DOI: $10.1039 / \mathrm{c} 8 \mathrm{ra0} 0223 \mathrm{c}$

rsc.li/rsc-advances

\section{Germanium quantum dot/nitrogen-doped graphene nanocomposite for high-performance bulk heterojunction solar cells $\uparrow$}

\author{
Tabitha A. Amollo, ${ }^{a}$ Genene T. Mola ${ }^{b}$ and Vincent O. Nyamori (D) *a
}

This study presents the successful synthesis of a novel nanocomposite, namely a germanium quantum dot/ nitrogen-doped graphene nanocomposite (GeQD/NGr), and its use in the modification of the photoactive medium of bulk heterojunction solar cells (BHJ-SCs). The nanocomposite was prepared in two sequential steps. Firstly, a reduced graphene oxide-germanium oxide nanocomposite $\left(\mathrm{rGO}-\mathrm{GeO}_{2}\right)$ was synthesized by microwave-assisted solvothermal reaction. The second step involved simultaneous $\mathrm{N}$-doping of graphene and reduction of $\mathrm{GeO}_{2}$ to obtain the GeQD/NGr nanocomposite by thermal treatment. The nanocomposite consists of highly crystalline, spherical shaped GeQDs with a mean diameter of $4.4 \mathrm{~nm}$ affixed on the basal planes of NGr sheets. Poly-3-hexylthiophene (P3HT), (6-6)phenyl-C60-butyric acid methyl ester (PCBM) and GeQD/NGr were used as the photoactive layer blend in the fabrication of BHJ-SCs. Enhanced short-circuit current density $\left(J_{s c}\right)$ and fill factor (FF) is derived from the incorporation of the GeQD/NGr nanocomposite in the active layer. The nanocomposite in the active layer blend serves to ensure effective charge separation and transportation to the respective electrodes. Consequently, an improvement of up to $183 \%$ in the power conversion efficiency is achieved in the BHJ-SCs by the GeQD/NGr modification.

\section{Introduction}

Photovoltaics is a promising solution to the energy and associated environmental concerns of the $21^{\text {st }}$ century. Organic photovoltaics (OPV) offer the advantages of low cost of materials and production, simple solution-based processing, light weight and flexibility. Currently, some high-efficiency polymer solar cells (PSCs) are based on a blend of an electron rich donor polymer and a fullerene derivative as the acceptor material. In such devices, the donor polymer and the fullerene are intermixed together in a blend to form a bulk heterojunction (BHJ) structure. One of the main factors that limits the power conversion efficiency (PCE) of OPVs is their very low exciton diffusion length $c a$. $10 \mathrm{~nm} .{ }^{1,2}$ The $\mathrm{BHJ}$ device structure alleviates this challenge by the formation of the excitons within the exciton diffusion length of the active layer blend. ${ }^{3}$ This ensures effective charge separation for better device PCE. ${ }^{4}$ The most popular conjugated polymer, poly-3-hexylthiophene (P3HT) and its fullerene counterpart, (6-6) phenyl-C61-butyric acid methyl ester (PCBM) forms the commonly used active layer blend. Compared to the traditional silicon-based solar cells, OPVs such as those based on

${ }^{a}$ University of KwaZulu-Natal, Westville Campus, School of Chemistry and Physics, Private Bag X54001, Durban 4000, South Africa.E-mail: Nyamori@ukzn.ac.za

${ }^{b}$ University of KwaZulu-Natal, Pietermaritzburg Campus, School of Chemistry and Physics, Private Bag X01, Scottsville, 3209, South Africa

† Electronic supplementary information (ESI) available. See DOI: $10.1039 / \mathrm{c} 8 \mathrm{ra04223c}$
P3HT:PCBM suffer from low short-circuit current density $\left(J_{\text {sc }}\right)$ which mainly originate from their relatively narrow spectral absorption $^{5}$ and low carrier mobility. ${ }^{6,7}$ Hence, one would consider tailoring the optical and electronic properties of the active layer materials to harvest most of the solar energy, generate high number of charge carriers, and transport the charge carriers to the outside circuitry with minimal recombination. Another effective strategy towards improved PCE in PSCs is the formation of favourable active layer film morphology for exciton dissociation and charge transport. This serves to mitigate both geminate and non-geminate charge carrier recombination processes. ${ }^{\mathbf{8 - 1 0}}$ Graphene has been under intense investigation to enhance charge generation and transportation within the photoactive medium of PSCs.

Pristine graphene is a one-atom-thick, 2D nanomaterial with $\mathrm{sp}^{2}$-hybridized carbon atoms which are packed in a honeycomb lattice structure. It exhibits outstanding properties such as high electrical conductivity, carrier mobility, specific surface area, optical transparency, mechanical strength and flexibility. ${ }^{\mathbf{1 1}}$ Owing to these unique material characteristics, graphene and/ or its derivatives are suited for application in solar cells as a transparent conductive electrode,${ }^{12}$ anode ${ }^{13,14}$ or anode buffer layer ${ }^{\mathbf{1 5}}$ and electron transport layer. ${ }^{\mathbf{1 6}}$ Nevertheless, pristine graphene lacks an energy band gap which is a requisite in most of the applications involving electronic transport. For this reason, research studies with the aim of opening a band gap thereby suiting graphene for electronic structure-related applications, have been on going. Advantageously, graphene affords 
numerous possibilities for tailoring its carbon backbone structure and hence, its physicochemical properties. For example, heteroatom-doping and chemical modification are potent means of band structure modulation in graphene. ${ }^{17,18}$ By heteroatom doping, carbon atoms of graphene are either substituted by the dopant atoms or the two interact through covalent bonding. ${ }^{19}$

$\mathrm{N}$-doping of graphene has been an attractive topic of research for tuning the electronic band structure of graphene. This route is plausible since $\mathrm{N}$ has similar atomic size to carbon and may not significantly distort the planar structure of graphene. ${ }^{20}$ Also, $\mathrm{N}$ has a strong electron withdrawing capability ${ }^{21}$ and can form strong covalent bonds with the $\mathrm{C}$ atoms resulting in a stable structure. ${ }^{22}$ Consequently, band structure modulation viz. band gap opening in the energy spectrum and shifting of the Fermi level $\left(E_{\mathrm{F}}\right)$ position in graphene is attainable by Ndoping. Substitutional N-doping of graphene endows it with an n-type semiconducting behaviour with the $E_{\mathrm{F}}$ shifting above the Dirac point and a suppressed density of states (DOS) near the $E_{\mathrm{F}}$ to create a band gap. ${ }^{17,23-25}$ Moreover, N-doping provides nucleation sites for further functionalization of the graphene back bone. ${ }^{26}$ Different bonding configurations of N-dopant to C, also known as $\mathrm{N}$-species, evolve from substitutional $\mathrm{N}$-doping of graphene. The three major $\mathrm{N}$-species are pyridinic- $\mathrm{N}$, pyrrolic- $\mathrm{N}$ and quaternary- $\mathrm{N}$ (graphitic- $\mathrm{N}$ ). Pyridinic- $\mathrm{N}$ and pyrrolic- $\mathrm{N}$ contribute one and two p-electrons, respectively, to the $\pi$-electron network of graphene. ${ }^{26,27}$ In terms of the position of the Nspecies in the graphene lattice, pyridinic-N bonds with two $\mathrm{C}$ atoms at the edges while pyrrolic- $\mathrm{N}$ bonds in to form a fivemembered ring structure. In the case of quaternary- $\mathrm{N}$, it is characterized by substitution of $\mathrm{C}$ atoms in the hexagonal ring by $\mathrm{N}$ atoms. ${ }^{2,26}$ When classification is based on the hybridization system, pyridinic- $\mathrm{N}$ and quaternary- $\mathrm{N}$ form $\mathrm{sp}^{2}$ hybridization system while pyrrolic-N is $\mathrm{sp}^{3}$-hybridized. ${ }^{26,27}$ Consequently, the type of $\mathrm{N}$-species formed influence the properties of graphene modulated. Thus, N-doping of graphene extends its functionality to various applications such as electronics, solar cells, capacitors, batteries, sensors etc. ${ }^{19,26} \mathrm{~N}$ doped graphene in the active layer have been shown to enhance charge selectivity and transportation in $\mathrm{BHJ}$ organic solar cells (OSCs). ${ }^{6}$ Some of the methods used for N-doping of graphene include chemical vapor deposition (CVD), arc discharge, solvothermal method, thermal treatment, plasma treatment and wet chemical methods. ${ }^{19,27}$

Germanium is one of the leading semiconductors in electronics and optoelectronics technologies. Its large Bohr exciton radius, $c a .24 .3 \mathrm{~nm}$, leads to significant quantum size effects. ${ }^{28}$ The size-tunability of the optical and electrical characteristics of Ge makes it amenable for novel strategies aimed at efficient conversion of photons to electrons. ${ }^{29} \mathrm{Ge}$ is characterized by a narrow band gap ca. $0.67 \mathrm{eV}$, high carrier mobility and high absorption coefficient ca. $2.0 \times 10^{5} \mathrm{~cm}^{-1}$ at $2 \mathrm{eV} \cdot{ }^{30}$ Moreover, Ge is non-toxic and environmentally friendly in contrast to semiconductors consisting of $\mathrm{Cd}, \mathrm{Pb}$ or $\mathrm{Hg}^{.}{ }^{31}$ Because of these properties, Ge nanoparticles/nanostructures are viable for application in photodetectors, solar cells, lithium ion batteries and biological imaging. ${ }^{32} \mathrm{Ge}$ is one of the leading semiconductors in photovoltaics. ${ }^{33,34}$ For example, when used as a bottom-element in solar cells, Ge aids the optical absorption in the infrared region of the solar spectrum thus, extending the spectral absorption of these devices for improved PCE. ${ }^{34}$ Similarly, Ge has been shown to broaden the absorption spectrum of P3HT. ${ }^{35}$ Furthermore, the fact that colloidal Ge nanoparticles are dispersible in to solution allows for cost-effective solutionbased device production.

Commercial application of graphene and/or its derivatives requires processing techniques that are compatible with large scale, low-cost and environment friendly productivity. In this regard, liquid-phase exfoliation of graphene oxide (GO) comes in handy. GO is oxygen functionalized graphene sheet consisting of the hydroxyl and epoxy groups on the basal planes and the carboxylic groups on the edges. ${ }^{36}$ In GO, the conjugation of the $\mathrm{sp}^{2}$ carbon network is disrupted by the $\mathrm{C}-\mathrm{O}$ bonds rendering it an insulator. The electronic structure of graphene can be restored in GO by either thermal or chemical reduction. Nevertheless, achieving GO-based materials with well-defined electronic structure for optimized device performance in various applications remains a challenge. For application in the active layer of OPV, graphene-based materials should be designed in such a way as to enhance charge separation and transportation to the electrodes with minimal recombination. Thus, these materials should not only increase the electrical conductivity of the polymer medium but also ensure ideal charge selectivity. In this study, we report the use of a novel graphene-based nanomaterial, germanium quantum dot/ nitrogen-doped graphene nanocomposite (GeQD/NGr), in the active layer of bulk heterojunction solar cells (BHJ-SCs). This study sought to explore the synergistic effect of the unique properties of $\mathrm{N}$-doped graphene and $\mathrm{Ge}$ on the photovoltaic performance of BHJ-SCs. Devices with GeQD/NGr in the active layer were fabricated and characterized for photovoltaic performance. Additionally, a reference device based on P3HT:PCBM was prepared and analysed. The impact of the nanocomposite on the photovoltaic performance of the solar cells is evaluated and discussed in detail.

\section{Experimental section}

\subsection{Synthesis and characterization of germanium quantum} dot/nitrogen-doped graphene nanocomposite

GO was prepared by the modified Hummers method..$^{37}$ Reduced graphene oxide-germanium oxide nanocomposite ( $\left.\mathrm{rGO}-\mathrm{GeO}_{2}\right)$ was synthesized by the microwave-assisted solvothermal reaction as outlined by Li et al. ${ }^{38}$ and Amollo et al. ${ }^{36}$ Typically, in the synthesis, GO (0.5 g) was dispersed in anhydrous ethanol (50 $\mathrm{ml})$ by ultrasonication. In a different set up, germanium tetrachloride $(1.0 \mathrm{~g})$ was stirred in anhydrous ethanol $(5 \mathrm{ml})$. The two solutions were mixed together and stirred further for 20 minutes. After which, the obtained solution was irradiated by microwaves at $180{ }^{\circ} \mathrm{C}$. From the microwave synthesis, a black colloidal suspension composed of $\mathrm{rGO}-\mathrm{GeO}_{2}$ nanocomposite was collected. This was thoroughly washed with ethanol and double distilled water and then dried in a vacuum oven. To

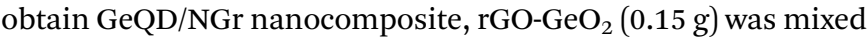


with 2-methylimidazole ( $0.4 \mathrm{~g})$ and ultrasonicated in absolute ethanol $(100 \mathrm{ml})$ for $1 \frac{1}{2} \mathrm{hrs}$. The obtained solution was heated under stirring at $80^{\circ} \mathrm{C}$ for $12 \mathrm{~h}$. This was followed by drying in the vacuum oven at $70^{\circ} \mathrm{C}$. The as-obtained solid was placed in a crucible and subjected to thermal treatment in a tube furnace under the flow of $10 \%$ hydrogen-argon gas. The samples were heated at $700{ }^{\circ} \mathrm{C}$ for $2 \mathrm{~h}$ at a rate of $5{ }^{\circ} \mathrm{C} \mathrm{min}{ }^{-1}$. Finally, GeQD/ NGr nanocomposite was collected from the tube furnace. For comparison purposes, N-doped graphene (NGr) was prepared by thermal treatment following the same protocol, as opposed to the microwave approach, GO $(0.15 \mathrm{~g})$ and 2-methylimidazole $(0.4 \mathrm{~g})$ were used as the starting materials in the latter approach.

The structural and morphological properties of the nanocomposite and NGr were analysed by transmission electron microscopy (TEM: JEOL JEM 1010), field emission scanning electron microscopy (SEM: JEOL JSM 6100) and high-resolution transmission electron microscopy (HR-TEM: JEOL JEM 2100). The crystalline phases of the samples were analysed by powder X-ray diffraction carried out using a Bruker D8 Advance X-ray powder diffractometer with high-intensity $\mathrm{Cu} \mathrm{K} \alpha$ radiation $(\lambda=0.15406 \mathrm{~nm})$. The chemical structures of the samples were analysed with a $100 \mathrm{~mW}$ DeltaNu Advantage 532 spectrometer (laser wavelength of $532 \mathrm{~nm}$ ). X-ray photoelectron spectroscopy (XPS) analysis was carried out using a Kratos Axis Ultra with an $\mathrm{Al}$ anode at a $\mathrm{HT}$ anode voltage of $15 \mathrm{kV}$, an emission current of $10 \mathrm{~mA}$ and $160 \mathrm{eV}$ scan resolution. Thermal stability of the sample was measured with a $\mathrm{Q}$ series ${ }^{\mathrm{TM}}$ Thermal Analyzer DSC/TGA (Q600). The surface area and porosity of the nanocomposite was obtained from a Micrometrics TriStar II surface area and porosity analyser.

\subsection{Device fabrication and characterization}

To obtain the active layer solution, $\mathrm{P} 3 \mathrm{HT}: \mathrm{PC}_{60} \mathrm{BM}$ at ratio $1: 1$ and GeQD/NGr nanocomposite at 3 and $5 \mathrm{wt} \%$ were prepared in chloroform at $20 \mathrm{mg} \mathrm{ml}^{-1}$ concentration. NGr at $3 \mathrm{wt} \%$ was also used in the active layer blend for comparison. The active layer solution was stirred for $4 \mathrm{~h}$ at $40{ }^{\circ} \mathrm{C}$. Devices were fabricated in the BHJ architecture of glass/ITO/PEDOT:PSS/P3HT:PCBM:NGr-Ge QDs/LiF/Al as shown in Fig. 1. Indium tin oxide (ITO) coated glass substrates, of $15 \Omega /$ sq sheet resistance, were etched in an acid solution. This was followed by sequential ultrasonication in deionized water, acetone and isopropanol for $10 \mathrm{~min}$ each. The substrates were then dried in a vacuum oven at $120{ }^{\circ} \mathrm{C}$ for 30 min. Poly(3,4-ethylenedioxythiophene):poly(styrenesulfonate) (PEDOT:PSS) was spin-coated on the substrates at $4500 \mathrm{rpm}$ for $60 \mathrm{~s}$ and then dried in the vacuum oven at $120{ }^{\circ} \mathrm{C}$ for $20 \mathrm{~min}$. Thereafter, spin coating of the active layer solution was done at 1200 rpm for $30 \mathrm{~s}$ on the PEDOT:PSS layer. The samples were then thermally annealed in a tube furnace at $120^{\circ} \mathrm{C}$ for 5 min under the flow of nitrogen gas. Eventually, $\mathrm{LiF}(0.4 \mathrm{~nm})$ and $\mathrm{Al}$ electrode (50 $\mathrm{nm}$ ) were thermally deposited on to the samples under a vacuum at $10^{-6}$ mbar base pressure. The current-voltage $(J-V)$ measurements for the devices were done using a Keithley HP2400 sourcemeter and a solar simulator (model SS50AAA) under air-mass 1.5 global (A.M 1.5G) illumination and integrated power intensity of $100 \mathrm{~mW} \mathrm{~cm}^{-2}$. The optical absorption spectra of the active layer films were acquired from a Raleigh 1601 UV-vis-NIR spectrophotometer while the films morphology was analysed by SEM. The fabrication and characterization of the devices was carried out in an ambient environment and the effective area for the devices was $4 \mathrm{~mm}^{2}$.

\section{Results and discussion}

\subsection{Germanium quantum dot/nitrogen-doped graphene nanocomposite}

GeQD/NGr nanocomposite was prepared in two subsequent steps as illustrated schematically in Fig. 2. rGO-GeO $\mathrm{G}_{2}$ nanocomposite was first synthesized via the microwave-assisted

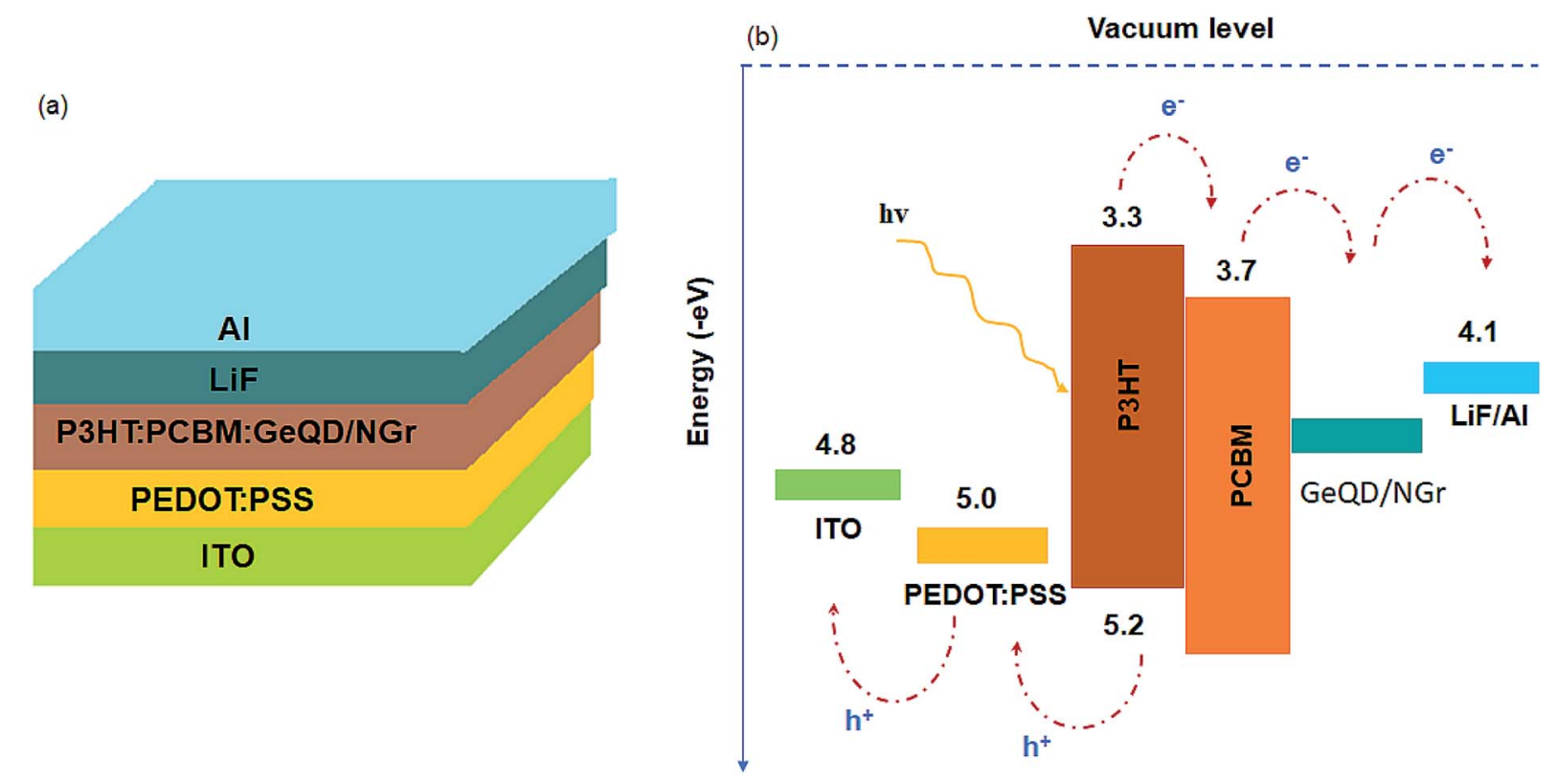

Fig. 1 (a) Schematic of the BHJ-SCs with GeQD/NGr nanocomposite in the active layer and (b) energy level diagram of the device materials. 


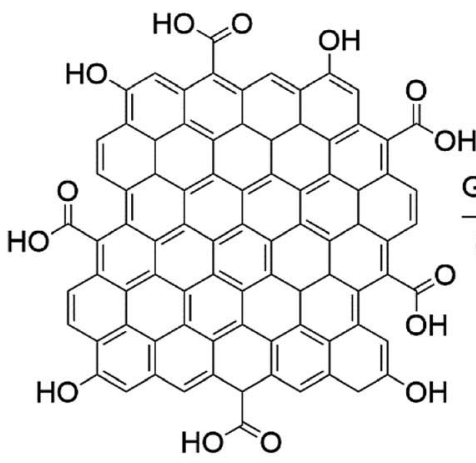

GO

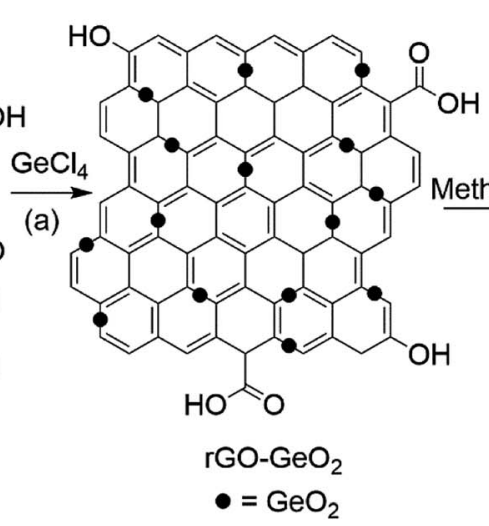

(b)

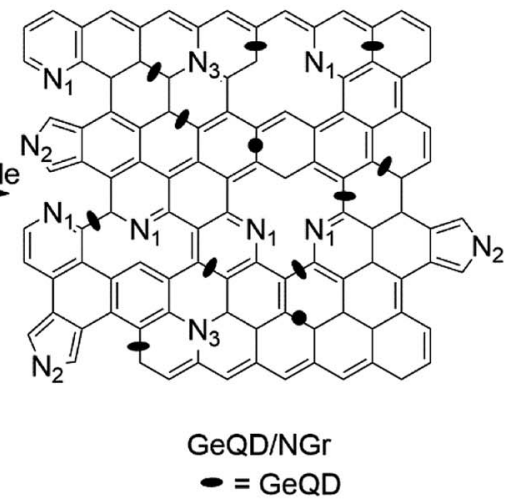

$\mathrm{N}_{1}=$ Pyridinic, $\mathrm{N}_{2}=$ Pyrollic, $\mathrm{N}_{3}=$ Graphitic

Fig. 2 Schematic illustration for the preparation of $\mathrm{GeQD} / \mathrm{NGr}$ nanocomposite: (a) microwave-assisted solvothermal synthesis of $\mathrm{rGO}-\mathrm{GeO}_{2}$ and (b) thermal treatment of $\mathrm{rGO}-\mathrm{GeO}_{2}$ composite with methylimidazole.

solvothermal reaction using $\mathrm{GO}$ as the starting material. Reduction of $\mathrm{GO}$ and formation of $\mathrm{rGO}-\mathrm{GeO}_{2}$ nanocomposite was simultaneously achieved in this procedure. In the second step, $\mathrm{rGO}-\mathrm{GeO}_{2}$ was used as the starting material to prepare $\mathrm{GeQD} / \mathrm{NGr}$ nanocomposite by thermal treatment. At this stage, $\mathrm{N}$-doping of graphene and formation of the GeQD/NGr nanocomposite were concurrently realized (Fig. 3).

The TEM and SEM images of the obtained nanocomposite are shown in Fig. S1 $\uparrow$ and 3, respectively. Clearly observed from the TEM image are transparent sheets rich in wrinkles and folds with edges characterized by scrolls. The TEM image also shows Ge QDs of uniform distribution attached on the NGr sheets. The surface morphology of the nanocomposite, as observed from the SEM image, is characterized by exfoliated sheets of good lamellar structure; these sheets are also rich in wrinkles. The TEM image of NGr (Fig. S2 $\dagger$ ) is characterized by folds and scrolls while its SEM image (Fig. S3†) shows aggregated sheets that are rich in wrinkles. Observed from the HR-TEM image of the nanocomposite (Fig. 4), are uniformly distributed Ge QDs anchored on the NGr sheets; these sheets are multi-layered in nature. The size of the Ge QDs is in the range of 2.0-10.6 nm. Inset of Fig. 4 is a histogram that shows the nanoparticles size distribution. A

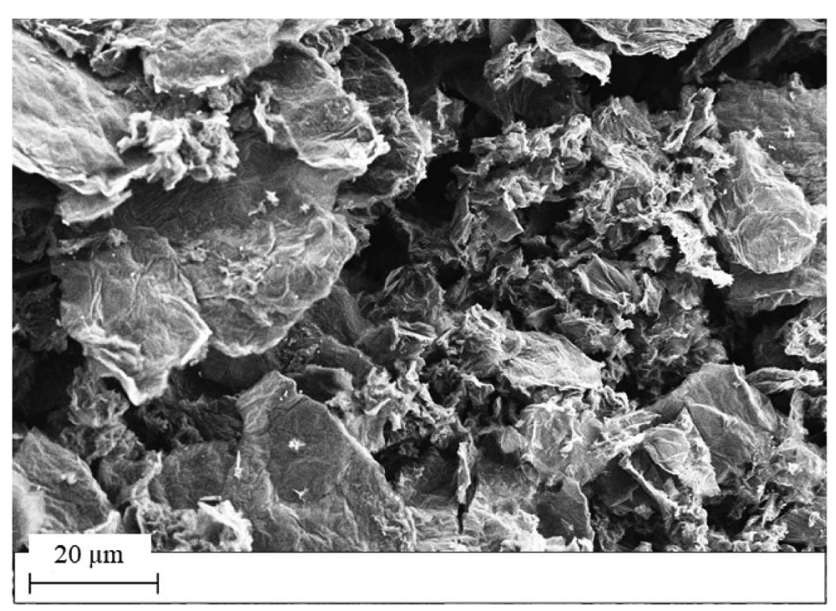

Fig. 3 SEM image of the GeQD/NGr nanocomposite. mean diameter of $4.4 \mathrm{~nm}$ with a standard deviation of $0.3 \mathrm{~nm}$ for the Ge QDs was obtained by performing a Gaussian fit to the histogram. Fig. S4 $\uparrow$ shows lattice fringes with $d$-spacing values of $0.34 \mathrm{~nm}$ and $0.26 \mathrm{~nm}$. The $0.34 \mathrm{~nm} d$-spacing is typical of NGr while $0.26 \mathrm{~nm}$ corresponds to the (201) plane of the diamond phase of cubic Ge. The crystal structure of the nanocomposite as characterized by XRD is illustrated in Fig. S5. $\dagger$ The diffraction peaks at $26^{\circ}, 47^{\circ}$ and $56^{\circ}$ are indexed to the (111), (220) and (311) planes of the diamond Ge cubic phase, respectively (JCPDS card no. 04-0545). The XRD pattern of NGr (Fig. S6 $\dagger$ ) is characterized by a sharp diffraction peak at $26^{\circ}$ and a small one at $43^{\circ}$. Thus, in the XRD pattern of the nanocomposite, the NGr peak at $26^{\circ}$ overlaps with that of (111) crystal plane of Ge.

Raman spectroscopy was used to evaluate the microstructure of the nanocomposite given its sensitivity to the crystalline

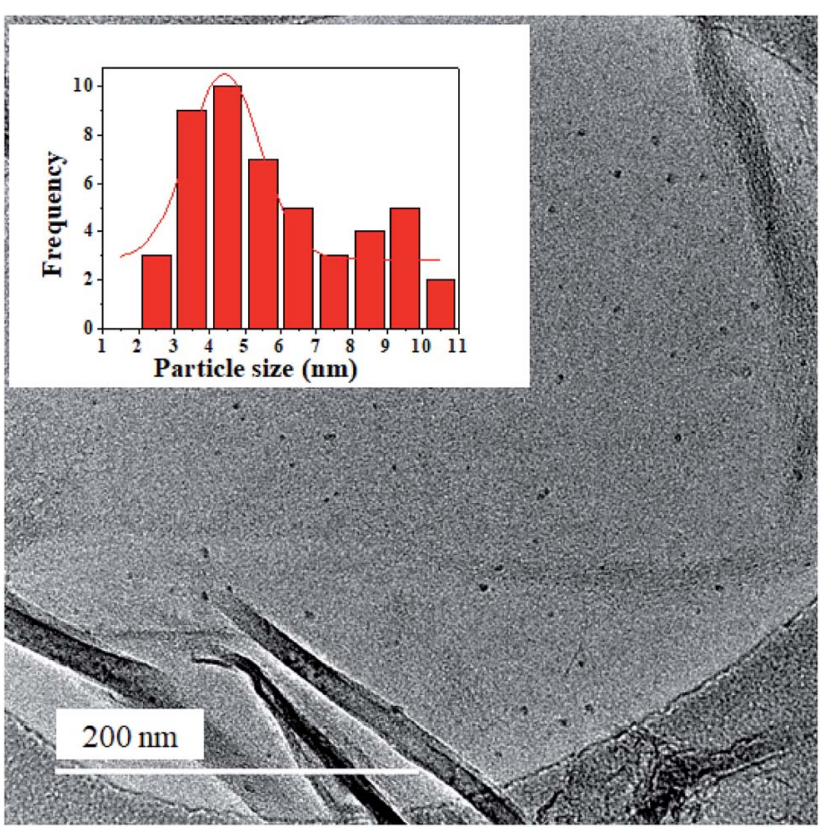

Fig. 4 HR-TEM image of the GeQD/NGr nanocomposite; inset is the Ge QDs size distribution histogram. 
state, defects and disorder in carbon-based samples. The Raman spectrum of the GeQD/NGr nanocomposite given in Fig. S7† manifests two peaks at 1355 and $1598 \mathrm{~cm}^{-1}$ corresponding to the D-band and G-band, respectively. The D-band is a signature of defects and disorder in the graphitic structure while the G-band is ascribed to the stretching vibrations of $\mathrm{sp}^{2}$ hybridized carbon atoms. The Raman spectrum of GO is also given in Fig. S7; $\uparrow$ the D-band and G-band for GO occurs at 1345 and $1585 \mathrm{~cm}^{-1}$, respectively. Thus, the nanocomposite manifest upward shifts in both the D-and G-bands peak position. A shift in the G-band peak position can be effected by doping, ${ }^{39}$ defects $^{\mathbf{4 0}}$ and strains. ${ }^{\mathbf{4 1}}$ The structural defects include boundaries, vacancies and heteroatoms in the graphitic lattice structure. ${ }^{42}$ Hence, the observed shift is indicative of charge transfer between the nanocomposite constituents i.e. NGr and Ge. Previous studies on NGr showed downshift of the G-band peak position given the electron-donating capability of $\mathrm{N}$ to graphene. ${ }^{6,43}$ Thus, the upward shift observed for the
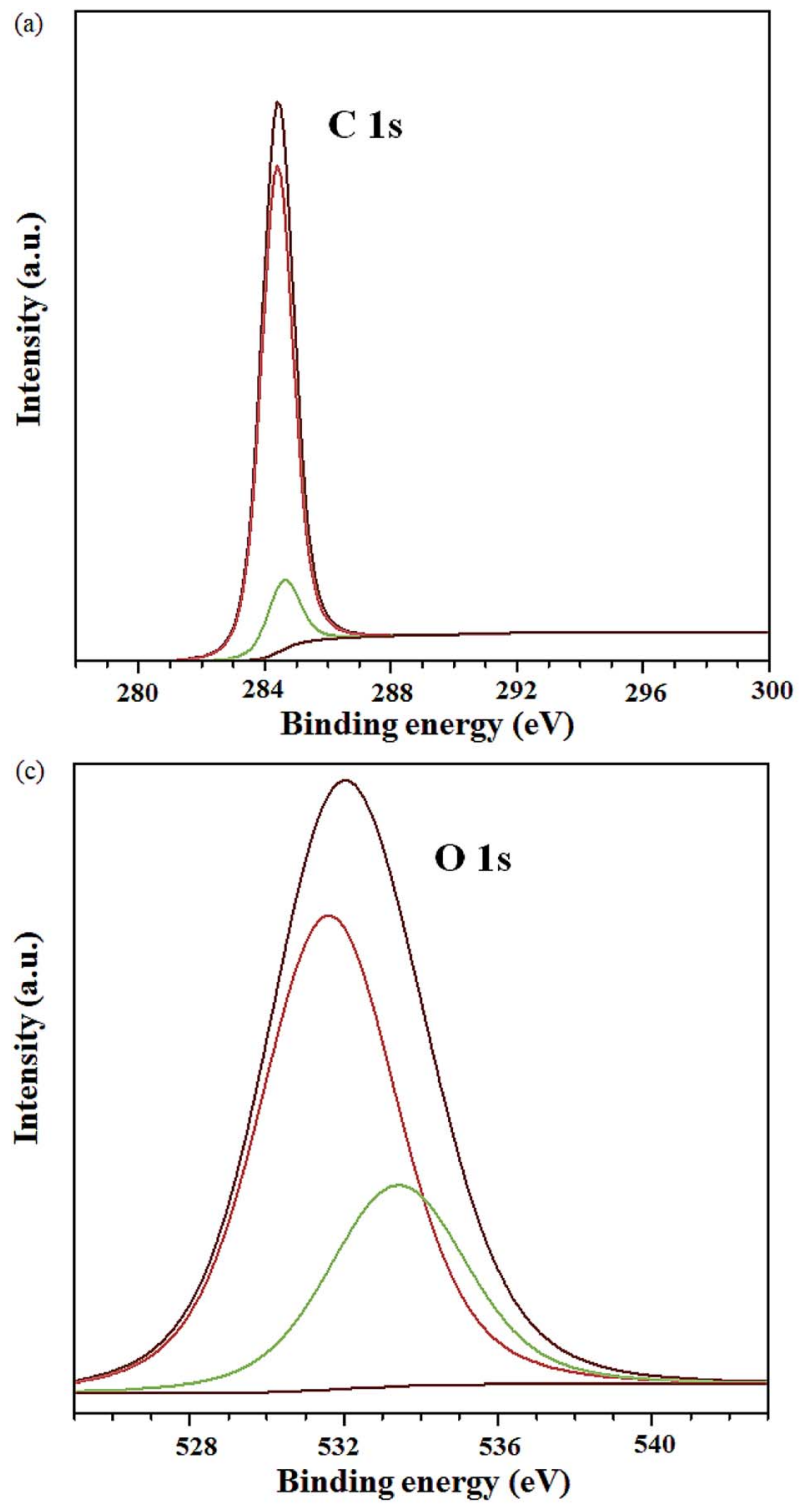

nanocomposite implies electron transfer from NGr to Ge. The well-documented Tuinstra-Koenig relation, ${ }^{\mathbf{4 4}} I_{\mathrm{D}} / I_{\mathrm{G}}$, which is a ratio of the integrated area of the D-to G-peaks was obtained for the nanocomposite. This ratio is a signature of the crystalline state of carbon-based samples. The nanocomposite exhibited an $I_{\mathrm{D}} / I_{\mathrm{G}}$ value of 1.19 which is higher than that of GO ca. 0.79. This implies the creation of defects in the graphitic structure upon $\mathrm{N}$ and Ge doping of graphene.

XPS analysis was carried out to evaluate the elemental composition of the nanocomposite, as well as, the respective chemical states. Fig. 5 shows the XPS spectra for the individual elements ( $\mathrm{C}, \mathrm{N}, \mathrm{O}$ and $\mathrm{Ge}$ ) in the nanocomposite. The atomic percentage of $\mathrm{C}, \mathrm{N}, \mathrm{O}$ and Ge was estimated as 95.7, 1.2, 2.6 and $0.5 \%$, respectively. The low doping level of $\mathrm{N}$ is attributed to the high reaction temperature which can break some of the $\mathrm{C}-\mathrm{N}$ bonds. ${ }^{42,45}$ Curve fitting of the $\mathrm{C}$ 1s spectrum (Fig. 5a) mainly yields two peaks at 284.4 and $284.6 \mathrm{eV}$; these are ascribed to the $\mathrm{sp}^{2}$ hybridized $\mathrm{C}$, implying that the carbon atoms in the
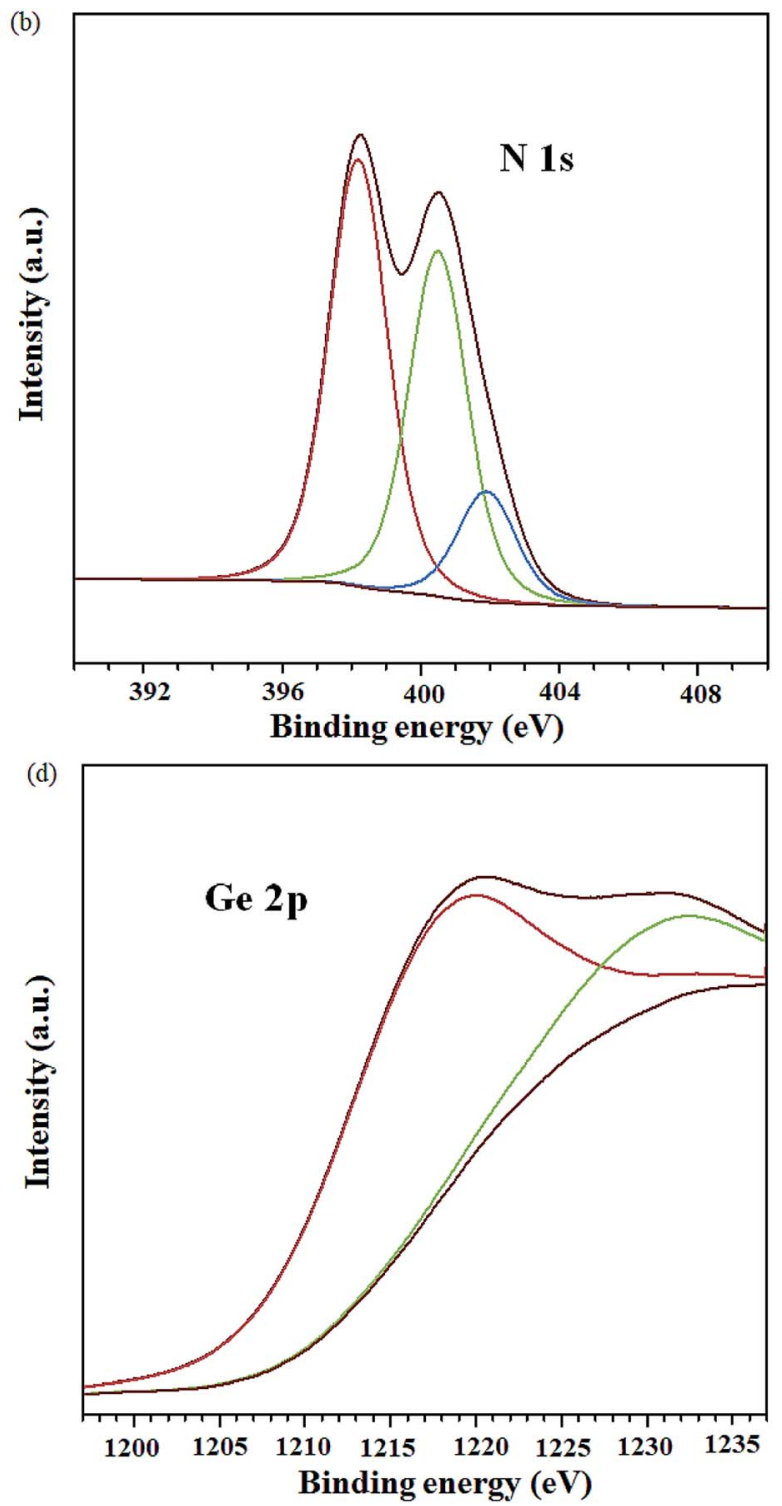

Fig. 5 XPS spectra for (a) C 1s, (b) N 1s, (c) $O$ 1s and (d) Ge 2p of GeQD/NGr nanocomposite. 
nanocomposite are predominantly packed in a honeycomb lattice structure. ${ }^{\mathbf{4 3 , 4 6}}$ Deconvolution of the Ge 2 p spectrum yielded two peaks at 1217.4 and $1230.6 \mathrm{eV}$ (Fig. 5d) corresponding to zero-valence $\mathrm{Ge}(78.2 \%)$ and $\mathrm{Ge}-\mathrm{O}$ bonds $(21.8 \%)$, respectively. Similarly, two components form the $\mathrm{O}$ 1s spectrum (Fig. 5c) i.e. peaks at 531.6 and $533.4 \mathrm{eV}$, corresponding to the $\mathrm{C}=\mathrm{O}$ and $\mathrm{O}=\mathrm{C}-\mathrm{O}$ oxygen functional groups, respectively. ${ }^{42}$ The $\mathrm{N}$ 1s spectrum was deconvoluted to three main peaks at 398.2, 400.5 and $401.9 \mathrm{eV}$ (Fig. 5b) which are assigned to the pyridinic-, pyrrolic- and graphitic-nitrogen, respectively. ${ }^{47,48}$ Of the different $\mathrm{N}$ 1s species in the nanocomposite, the pyridinic- $\mathrm{N}$ constituted the highest percentage $c a .43 .8 \%$ followed by pyrrolic $\mathrm{N}$ at $35.4 \%$. The graphitic-nitrogen formed a lower percentage $c a .11 .4 \%$. The pyridinic-N results in a local deficiency in electron which implies a high electron affinity ${ }^{\mathbf{4 9}}$ whereas, the graphitic-N is known to provide delocalized electrons in the graphitic structure which enhances electroconductivity. ${ }^{43}$ The overall effect of the pyridinic-, pyrrolic- and graphitic-N in the honeycomb structure of graphene is improved electrical conductivity. ${ }^{50}$ This, taken together with the high electron affinity associated with the pyridinic-N and Ge, favours the use of the nanocomposite in the active layer of OSCs as it would promote charge selectivity and transport.

Thermogravimetric analysis (TGA) was used to determine thermal stability of the nanocomposite; this was carried out in air at a heating rate of $10{ }^{\circ} \mathrm{C} \mathrm{min}^{-1}$. The obtained TGA thermogram is given in Fig. S8. $\dagger$ As observed from the thermogram, the nanocomposite manifests good thermal stability up to temperatures as high as $850{ }^{\circ} \mathrm{C}$; this is advantageous for the temperature processing requirements of OSCs. The slight weight increment observed from $250{ }^{\circ} \mathrm{C}$ is attributed to the oxidation of $\mathrm{Ge}$ to $\mathrm{GeO}_{2}$. A similar trend has been observed for germanium-graphene nanosheets. ${ }^{51}$

The nitrogen gas absorption-desorption isotherm for the GeQD/NGr nanocomposite is given in Fig. S9. $\uparrow$ The observed isotherm is type IV implying that the nanocomposite is a mesoporous material. ${ }^{52}$ The isotherm is accompanied by a pronounced type $\mathrm{H} 2$ hysteresis loop between $0.4-1.0 P / P_{\mathrm{o}}$; this hysteresis is associated with the occurrence of capillary condensation in the mesopores. The pronounced hysteresis is indicative of a high number of mesopores with evenly distributed pore size within the nanocomposite structure. ${ }^{53}$ A surface area of $67 \mathrm{~m}^{2} \mathrm{~g}^{-1}$ was obtained by Brunauer-Emmett-Teller (BET) analysis. This corroborates with the observed SEM image which shows well exfoliated wrinkled sheets. The pore size and volume of the nanocomposite were $15 \mathrm{~nm}$ and $0.12 \mathrm{~cm}^{3} \mathrm{~g}^{-1}$, respectively.

\subsection{Device characterization}

3.2.1 Optical absorption. The optical absorbance spectra for the photoactive layers containing 3 and $5 \mathrm{wt} \%$ of GeQD/NGr nanocomposite are shown in Fig. 6. The optical absorption spectrum of the reference device is also included in the same Fig. 6. These spectra were acquired on the active layer, spin coated on the PEDOT:PSS hole transport layer. The active layer films with GeQD/NGr nanocomposite manifest relatively stronger absorption intensity in comparison to the reference

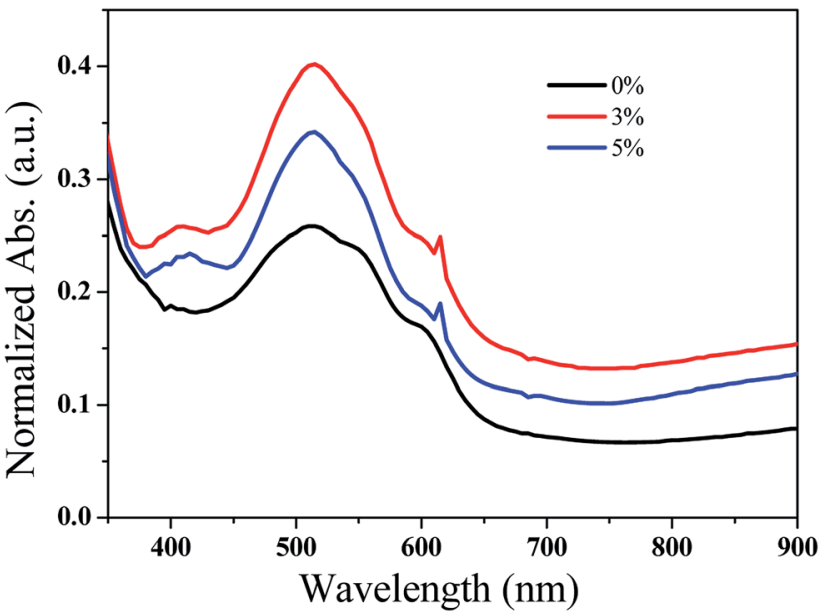

Fig. 6 UV-vis absorption spectra of the P3HT:PCBM:GeQD/NGr films at different GeQD/NGr concentration.

film. These spectra are characterized by an additional peak of low intensity between $380-440 \mathrm{~nm}$ and a sharp shoulder at $615 \mathrm{~nm}$. Hence, the inlay of the GeQD/NGr nanocomposite in the P3HT:PCBM blend not only improves its optical absorption but also broadens its absorbance spectrum to lower wavelengths.

3.2.2 Electrical properties. The performance of the fabricated BHJ-SCs was studied in terms of the measured electrical parameters such as the open-circuit voltage $\left(V_{\mathrm{oc}}\right), J_{\mathrm{sc}}$, fill factor $(\mathrm{FF})$, series resistance $\left(R_{\mathrm{s}}\right)$ and PCE. These parameters were deduced from the current-voltage $(J-V)$ characteristics of the devices taken under AM 1.5 illumination at integrated power intensity of $100 \mathrm{~mW} \mathrm{~cm} \mathrm{~cm}^{-2}$. For each sample, 12 devices were fabricated and tested. Some examples of the obtained $J-V$ plots are given in Fig. 7 while the best performance data are summarized in Table 1. Generally, the devices containing GeQD/NGr nanocomposite in the photoactive layer exhibited better photovoltaic performance in comparison to the P3HT:PCBM-based reference solar cell. The incorporation of the

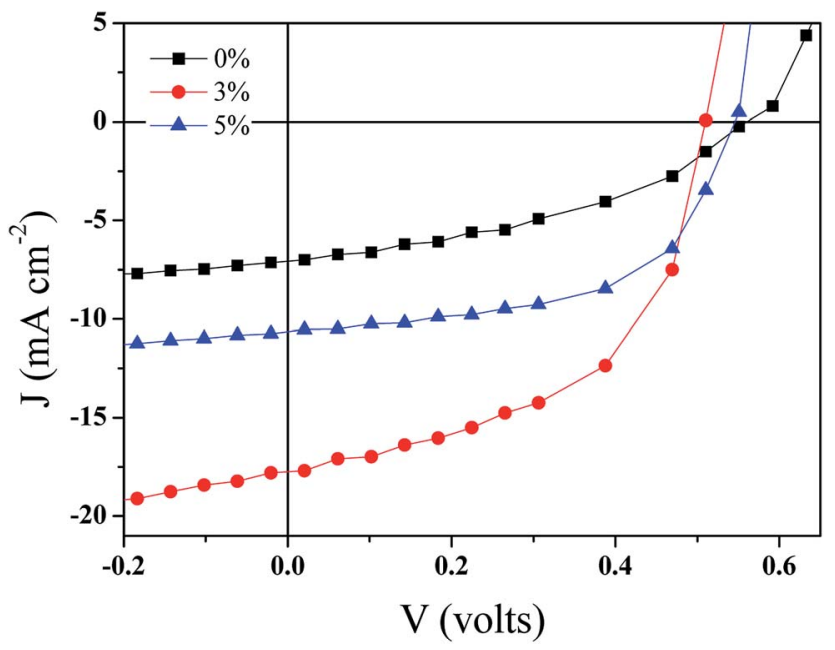

Fig. 7 The current-voltage characteristics of the $\mathrm{BHJ}-\mathrm{SCs}$ with GeQD/NGr in the active layer at different concentrations. 
Table 1 Electrical parameters of the fabricated devices

\begin{tabular}{llcrr}
\hline GeQD/NGr loading $(\mathrm{wt} \%)$ & $V_{\text {oc }}($ volts $)$ & $J_{\text {sc }}\left(\mathrm{mA} \mathrm{cm}^{-2}\right)$ & FF $(\%)$ & PCE $(\%)$ \\
\hline 0 & $0.56 \pm 0.006$ & $7.3 \pm 0.07$ & $45 \pm 0.7$ & $1.8 \pm 0.07$ \\
3 & $0.50 \pm 0.008$ & $18.9 \pm 0.28$ & $55 \pm 1.8$ & $5.1 \pm 0.12$ \\
5 & $0.56 \pm 0.01$ & $11.1 \pm 0.26$ & $54 \pm 1.3$ & $3.3 \pm 0.07$ \\
\hline
\end{tabular}

nanocomposite in the active layer results in improved $J_{\mathrm{sc}}$ and FF implying better charge carriers photogeneration and transportation to the electrodes. With regards to the concentration of the nanocomposite in the active layer, the device at $3 \mathrm{wt} \%$ of GeQD/NGr showed superior photovoltaic performance. It manifests a higher $J_{\mathrm{sc}}$ and lower $R_{\mathrm{s}}$ which is indicative of low non-geminate charge carrier recombination and thus, low charge carrier losses. On the other hand, the device with active layer at $5 \mathrm{wt} \%$ of the nanocomposite exhibit a relatively higher $R_{\mathrm{S}}$, in fact, comparable to the reference device. This corroborates with its relatively lower $J_{\mathrm{sc}}$ and implies the occurrence of higher charge carrier loss in the device through recombination processes. The FF of the devices with the active layer at 3 and $5 \mathrm{wt} \%$ of the nanocomposite are comparable but higher than that of the reference device. Since both devices have almost similar FF values, the charge carrier loss implied by the lower $J_{\mathrm{sc}}$ value of the device at $5 \mathrm{wt} \%$ of the nanocomposite is not solely due to a higher non-geminate recombination. This then bears the intimation that geminate recombination is higher in the device at $5 \mathrm{wt} \%$ of the nanocomposite. Generally, the improvement in both the $J_{\mathrm{sc}}$ and $\mathrm{FF}$ signifies better charge transport and collection upon incorporation of the nanocomposite in the active layer.

A large potential difference between the LUMO of the donor and the acceptor materials promotes charge separation in OSCs. Considering the work function of NGr ca. $-4.4 \mathrm{eV}$ (ref. 6) and that of Ge $c a .-4.78 \mathrm{eV}$ (ref. 54) in comparison to the LUMO level of P3HT $c a$. $-3.3 \mathrm{eV}$, a high potential difference is created in the active layer phases for effective exciton dissociation. Thus, geminate charge recombination is minimized in the devices. Moreover, because of the high electron affinity associated with the nanocomposite constituents, its incorporation in the active layer not only offers charge selectivity at the interfaces but also facilitates electrons transfer within the acceptor domains. The charge selectivity of the nanocomposite serves to ensure effective extraction of electrons hence, alleviates the recombination of holes and electrons. Another factor that affects both charge carriers separation and transportation is the nanoscale thin film morphology. Good crystallinity and miscibility of the donor and acceptor materials favours the formation of smooth morphology. Hence, the good crystalline quality of the GeQD/NGr nanocomposite is an additional advantage to its use in the active layer. Therefore, the inlay of GeQD/NGr nanocomposite in the active layer, at optimum concentration, facilitates exciton generation, dissociation and transport of the separated charge carriers to the outside circuitry for enhanced device performance.

3.2.3 Charge transport. To investigate the charge carrier transport phenomena in the fabricated devices, current-voltage measurements were done under dark conditions. Analysis of the space charge limited current (SCLC) regime was then done to obtain the charge carrier mobility. In the SCLC regime, charge transport in the system is determined by the inherent bulk material characteristics. The SCLC is usually described by the Mutt-Gurney law provided in eqn (1),

$$
J=9 / 8 \varepsilon \varepsilon_{0} \mu_{0} \exp \left(0.89 \gamma[V / L]^{1 / 2}\right) V^{2} / L^{3}
$$

where $\varepsilon$ is the relative permittivity, $\varepsilon_{0}$ is the vacuum permittivity, $\mu_{0}$ is the zero-field mobility, $\gamma$ is the field activation factor, $V$ is the applied voltage and $L$ is the active layer thickness. Fig. 8 shows the plots of the SCLC regime and fits to the curves according to Mutt-Gurney law. This law holds for the devices since the fits imbricate the actual curves as shown in the Fig. 8. The zero-field carrier mobility and field activation factor deduced from the fits to the SCLC plots for the devices are listed in Table 2. The devices with the GeQD/NGr nanocomposite in the active layer exhibit increased carrier mobilities by two orders of magnitude in comparison to the reference device. The improved carrier mobilities signifies an improved charge transport in the devices. Thus, the incorporation of the nanocomposite in the active layer results in the formation of interconnected pathways for charge transport. This serves to alleviate non-geminate charge recombination in the devices. The improved charge transport is evidenced by the higher $J_{\mathrm{sc}}$ values attained in the devices compared to the control device. The carrier mobilities of the devices at different nanocomposite concentration in the active layer are comparable and corroborates with their similar FF values.

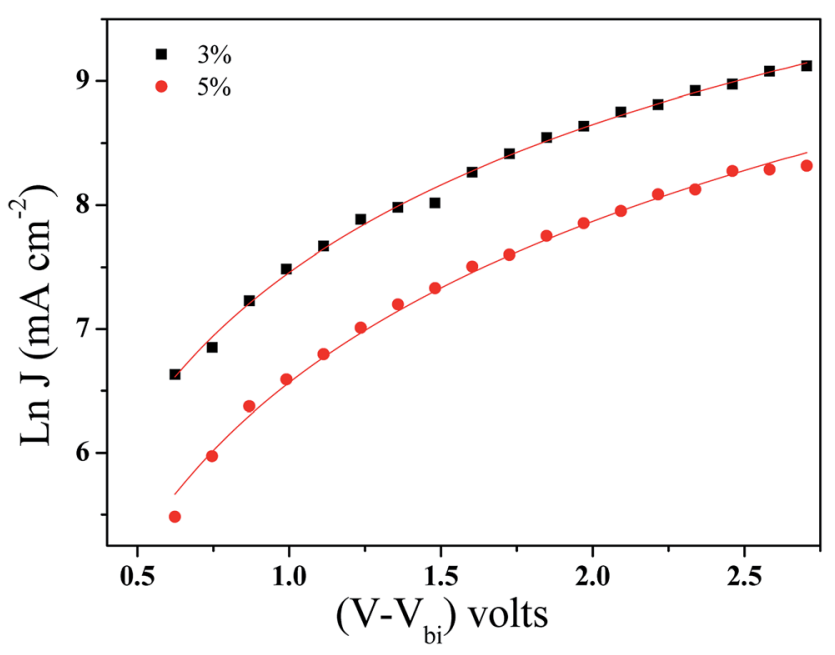

Fig. 8 Space charge limited currents of the BHJ-SCs with GeQD/NGr at different concentrations in the active layer; the solid lines are computer fits according to eqn (1). 
Table 2 Charge transport parameters of the BHJ-SCs with GeQD/ $\mathrm{NGr}$ in the active layer

\begin{tabular}{lll}
\hline GeQD/NGr loading $(\mathrm{wt} \%)$ & $\mu_{0}\left(\mathrm{~cm}^{2} \mathrm{~V}^{-1} \mathrm{~S}^{-1}\right)$ & $\gamma\left(\mathrm{cm} \mathrm{V}^{-1}\right)$ \\
\hline 0 & $3.96 \times 10^{-5}$ & $-1.9 \times 10^{-4}$ \\
3 & $4.81 \times 10^{-3}$ & $-1.8 \times 10^{-4}$ \\
5 & $1.53 \times 10^{-3}$ & $-8.0 \times 10^{-4}$
\end{tabular}

A solar cell with NGr at $3 \mathrm{wt} \%$ in the active layer was fabricated and analysed to elucidate the influence of Ge QDs in the photo active layer, on the photovoltaic performance. The obtained $J-V$ plot is shown in Fig. S10. $\dagger$ The device yielded a $V_{\text {oc }}$, $J_{\mathrm{sc}}, \mathrm{FF}, R_{\mathrm{s}}$ and PCE of $0.56 \mathrm{~V}, 10.5 \mathrm{~mA} \mathrm{~cm}{ }^{-2}, 50 \%, 768 \Omega$ and $2.9 \%$, respectively. Relative to the reference device, enhanced performance is achieved with NGr in the active layer, albeit, inferior to that of the GeQD/NGr modified devices. Specifically, the device manifests lower $J_{\mathrm{sc}}$ and higher $R_{\mathrm{s}}$ relative to the nanocomposite modified device implying a relatively higher charge carrier loss. With regards to the enhanced performance relative to the reference device, NGr offers charge selectivity and creates transport pathways for electron transfer hence, suppresses geminate charge recombination; this corroborates with the observation of Jun et al. ${ }^{6}$ Improved charge transport in the device is elucidated by charge transport analysis (Fig. S11 $\dagger$ ) which yielded a carrier mobility of $8.09 \times 10^{-4} \mu_{0}\left(\mathrm{~cm}^{2} \mathrm{~V}^{-1} \mathrm{~S}^{-1}\right)$ at a field activation factor of $2.0 \times 10^{-5} \gamma\left(\mathrm{cm} \mathrm{V}^{-1}\right)$; this is an order in magnitude higher than the reference device but lower than the nanocomposite modified devices in the same order. It is therefore evidenced that the incorporation of the Ge QDs in the active layer not only improves the charge selectivity, owing to its high electron affinity, but also adds impetus to the charge transport capability of the active layer blend.

\section{Conclusion}

In summary, we have demonstrated the influence of GeQD/NGr nanocomposite on the photovoltaic performance of BHJ-SCs. GeQD/NGr nanocomposite was synthesized by microwaveassisted solvothermal reaction followed by thermal treatment. TEM and HR-TEM analysis showed spherical shaped Ge QDS attached on the NGr sheets. Enhanced efficiency was achieved by the inclusion of the nanocomposite in the active layer. The improved PCE originated from enhanced $J_{\mathrm{sc}}$ and FF, giving the devices with nanocomposite in the active layer an edge over the reference device. With the nanocomposite in the active layer, a high electric field is built for effective charge separation. Also, given the high electron affinity of the nanocomposite's constituents, its inclusion in the active layer offers charge selectivity at the interface and aids in electron transfer within the acceptor domains. Charge transport analysis showed increased carrier mobilities for the modified active layer devices. The improved charge transport is attributed to the formation of interconnected pathways upon incorporation of the GeQD/NGr nanocomposite. Because of the additive conductive pathways for charge transfer, non-geminate charge recombination is suppressed in the devices. Therefore, the inclusion of GeQD/NGr nanocomposite in the active layer of $\mathrm{BHJ}$-SCs improves charge carriers generation, separation and transfer to the electrodes to yield an improvement in PCE by up to $183 \%$. The studies presented herein reveal the prospects of tuning the functionality of graphene for optimized performance in PSCs.

\section{Conflicts of interest}

There are no conflicts to declare.

\section{Acknowledgements}

The authors are grateful to the National Research Foundation (NRF), the University of KwaZulu-Natal and the UKZN Nanotechnology Platform for supporting this research work. The funding bodies did not participate in the research design, data collection and analysis, manuscript development or in the decision for its submission.

\section{References}

1 B. Kannan, K. Castelino and A. Majumdar, Nano Lett., 2003, 3, 1729-1733.

2 M. Sim, J. Shin, C. Shim, M. Kim, S. B. Jo, J.-H. Kim and K. Cho, J. Phys. Chem. C, 2014, 118, 760-766.

3 X. Wan, G. Long, L. Huang and Y. Chen, Adv. Mater., 2011, 23, 5342-5358.

4 W.-Q. Wu, D. Chen, F. Li, A. R. Pascoe, Y.-B. Cheng and R. A. Caruso, Nano Energy, 2017, 32, 187-194.

5 N. Gasparini, M. Salvador, T. Heumueller, M. Richter, A. Classen, S. Shrestha, G. J. Matt, S. Holliday, S. Strohm and H. J. Egelhaaf, Adv. Energy Mater., 2017, 22, 1701561.

6 G. H. Jun, S. H. Jin, B. Lee, B. H. Kim, W.-S. Chae, S. H. Hong and S. Jeon, Energy Environ. Sci., 2013, 6, 3000-3006.

7 L. Lu, M. A. Kelly, W. You and L. Yu, Nat. Photonics, 2015, 9, 491-500.

8 C. Liu, X. Hu, C. Zhong, M. Huang, K. Wang, Z. Zhang, X. Gong, Y. Cao and A. J. Heeger, Nanoscale, 2014, 6, 14297-14304.

9 S. O. Oseni and G. T. Mola, Sol. Energy, 2017, 150, 66-72.

10 W.-Q. Wu, D. Chen, Y.-B. Cheng and R. A. Caruso, Sustainable Energy Fuels, 2017, 1, 1960-1967.

11 T. A. Amollo, G. T. Mola, M. Kirui and V. O. Nyamori, Crit. Rev. Solid State Mater. Sci., 2018, 43, 133-157.

12 G. S. Selopal, R. Milan, L. Ortolani, V. Morandi, R. Rizzoli, G. Sberveglieri, G. P. Veronese, A. Vomiero and I. Concina, Sol. Energy Mater. Sol. Cells, 2015, 135, 99-105.

13 J. Cao, Y. Zhu, X. Yang, Y. Chen, Y. Li, H. Xiao, W. Hou and J. Liu, Sol. Energy Mater. Sol. Cells, 2016, 157, 814-819.

14 D. Joly, J.-W. Jung, I.-D. Kim and R. Demadrille, J. Mater. Chem. C, 2016, 4, 10173-10197.

15 H. P. Kim, A. R. bin Mohd Yusoff and J. Jang, Sol. Energy Mater. Sol. Cells, 2013, 110, 87-93.

16 R. Sharma, F. Alam, A. Sharma, V. Dutta and S. Dhawan, J. Mater. Chem. C, 2014, 2, 8142-8151. 
17 X. Fan, Z. Shen, A. Liu and J.-L. Kuo, Nanoscale, 2012, 4, 2157-2165.

18 W.-Q. Wu, D. Chen, R. A. Caruso and Y.-B. Cheng, J. Mater. Chem. A, 2017, 5, 10092-10109.

19 X. Wang, G. Sun, P. Routh, D.-H. Kim, W. Huang and P. Chen, Chem. Soc. Rev., 2014, 43, 7067-7098.

20 S. U. Lee, R. V. Belosludov, H. Mizuseki and Y. Kawazoe, Small, 2009, 5, 1769-1775.

21 B. Guo, Q. Liu, E. Chen, H. Zhu, L. Fang and J. R. Gong, Nano Lett., 2010, 10, 4975-4980.

22 M. Fan, Z.-Q. Feng, C. Zhu, X. Chen, C. Chen, J. Yang and D. Sun, J. Mater. Sci., 2016, 51, 10323-10349.

23 A. J. Marsden, P. Brommer, J. J. Mudd, M. A. Dyson, R. Cook, M. Asensio, J. Avila, A. Levy, J. Sloan and D. Quigley, Nano Res., 2015, 8, 2620-2635.

24 B. He, Z. Ren, C. Qi, S. Yan and Z. Wang, J. Mater. Chem. C, 2015, 3, 6172-6177.

25 S. H. Park, J. Chae, M.-H. Cho, J. H. Kim, K.-H. Yoo, S. W. Cho, T. G. Kim and J. W. Kim, J. Mater. Chem. C, 2014, 2, 933-939.

26 H. Wang, T. Maiyalagan and X. Wang, ACS Catal., 2012, 2, 781-794.

27 R. Yadav and C. Dixit, Journal of Science: Advanced Materials and Devices, 2017, 2, 141-149.

28 Y. Maeda, Phys. Rev. B, 1995, 51, 1658.

29 V. I. Klimov, Semiconductor and metal nanocrystals: synthesis and electronic and optical properties, CRC Press, New York, 2003.

30 D. Carolan and H. Doyle, J. Mater. Chem. C, 2014, 2, 35623568.

31 J. Fan and P. K. Chu, Small, 2010, 6, 2080-2098.

32 D. D. Vaughn II and R. E. Schaak, Chem. Soc. Rev., 2013, 42, 2861-2879.

33 Y. Kim, N. D. Lam, K. Kim, W.-K. Park and J. Lee, Sci. Rep., 2017, 7, 42693.

34 V. Sorianello, L. Colace, C. Maragliano, D. Fulgoni, L. Nash and G. Assanto, Opt. Mater. Express, 2013, 3, 216-228.

35 D. J. Xue, J. J. Wang, Y. Q. Wang, S. Xin, Y. G. Guo and L. J. Wan, Adv. Mater., 2011, 23, 3704-3707.
36 T. A. Amollo, G. T. Mola and V. O. Nyamori, Nanotechnology, 2017, 28, 495703.

37 W. S. Hummers Jr and R. E. Offeman, J. Am. Chem. Soc., 1958, 80, 1339.

38 D. Li, K. H. Seng, D. Shi, Z. Chen, H. K. Liu and Z. Guo, J. Mater. Chem. A, 2013, 1, 14115-14121.

39 A. Das, S. Pisana, B. Chakraborty, S. Piscanec, S. Saha, U. Waghmare, K. Novoselov, H. Krishnamurthy, A. Geim and A. Ferrari, Nat. Nanotechnol., 2008, 3, 210-215.

40 A. Das, B. Chakraborty and A. Sood, Bull. Mater. Sci., 2008, 31, 579-584.

41 M. Huang, H. Yan, T. F. Heinz and J. Hone, Nano Lett., 2010, 10, 4074-4079.

42 Z. Xing, Z. Ju, Y. Zhao, J. Wan, Y. Zhu, Y. Qiang and Y. Qian, Sci. Rep., 2016, 6, 26146.

43 D. Wei, Y. Liu, Y. Wang, H. Zhang, L. Huang and G. Yu, Nano Lett., 2009, 9, 1752-1758.

44 F. Tuinstra and J. L. Koenig, Chem. Phys., 1970, 53, 11261130.

45 A. Śliwak, B. Grzyb, N. Díez and G. Gryglewicz, Appl. Surf. Sci., 2017, 399, 265-271.

46 C. Botas, P. Alvarez, C. Blanco, R. Santamaría, M. Granda, M. D. Gutiérrez, F. Rodríguez-Reinoso and R. Menéndez, Carbon, 2013, 52, 476-485.

47 S. Kabir, K. Artyushkova, A. Serov, B. Kiefer and P. Atanassov, Surf. Interface Anal., 2016, 48, 293-300.

48 J. Pels, F. Kapteijn, J. Moulijn, Q. Zhu and K. Thomas, Carbon, 1995, 33, 1641-1653.

49 K. Share, A. P. Cohn, R. Carter, B. Rogers and C. L. Pint, ACS Nano, 2016, 10, 9738-9744.

50 A. Śliwak, N. Díez, E. Miniach and G. Gryglewicz, J. Appl. Electrochem., 2016, 46, 667-677.

51 C. Zhong, J.-Z. Wang, X.-W. Gao, D. Wexler and H.-K. Liu, J. Mater. Chem. A, 2013, 1, 10798-10804.

52 K. S. Sing, Pure Appl. Chem., 1985, 57, 603-619.

53 Z. Fan, Y. Liu, J. Yan, G. Ning, Q. Wang, T. Wei, L. Zhi and F. Wei, Adv. Energy Mater., 2012, 2, 419-424.

54 J. Dillon Jr and H. Farnsworth, J. Appl. Phys., 1957, 28, 174184. 